\title{
ON ORDINALS
}

BY W. V. QUINE AND HAO WANG

Communicated by A. M. Gleason, October 25, 1963

Ordinals in von Neumann's sense would naturally be defined by the transfinite recursion:

(1) $N O(x)$ for $(y)\left(S^{\prime \prime} y \subseteq y \cdot(z)\left(z \subseteq y \cdot \supset \cdot \cup_{z} \in y\right) \cdot \supset \cdot x \in y\right)$

were it not for the excessive demands on class existence for the values of ' $y$ '.

A trick of inversion (see [1]) obviated the need of infinite values of ' $y$ ' in the definition of natural numbers: give the role of $y$ to its complement $\bar{y}$ (without assuming existence of $\bar{y}$ ) and then reduce. I.e., put bars over the occurrences of ' $y$ ' after the quantifier and reduce. The same trick on (1) gives

(2) $N O(x)$ for $(y)\left(x \in y \cdot \bar{S}\right.$ “' $\left.y \subseteq y \cdot \supset(\exists z)\left(\cup_{z} \in y \cdot y \cap z=\Lambda\right)\right)$.

(2) and (1) are equivalent for naive set theory, since, taking $y$ as $\bar{y}$ in either, you get the other. But the superiority of (2) is that it requires, for each $x$, no $y$ bigger than $S^{\prime} x$.

Using the axioms of power set and Aussonderung, we can prove the law of transfinite induction:

$$
(x)\left(F x \supset F\left(S^{\prime} x\right)\right) \cdot(y)(y \subseteq F \cdot \supset F(U y)) \cdot N O(z) \cdot \supset F \mathbf{z} .
$$

Proof. By power set and Aussonderung, we can take $y$ in (2) as $\{x: x \subseteq z \cdot \sim F x\}$; so, by the last premise,

$z \subseteq z \cdot \sim F z \cdot(x)\left(S^{\prime} x \subseteq z \cdot \sim F\left(S^{\prime} x\right) \cdot \supset \cdot x \subseteq z \cdot \sim F x\right) \cdot \supset$

$$
(\exists y)(\mathrm{U} y \subseteq z \cdot \sim F(\cup y) \cdot \sim(\exists x)(x \subseteq z \cdot x \in y \cdot \sim F x)) .
$$

Dropping ' $z \subseteq z$ ' as true and ' $x \subseteq z$ ' as implied by ' $S$ ' $x \subseteq z$ ', and contraposing, we have:

$(x)\left(F x \cdot S^{\iota} x \subseteq z \cdot \supset F\left(S^{\prime} x\right)\right) \cdot$

$$
(y)(\mathrm{U} y \subseteq z \cdot(x)(x \subseteq z \cdot x \in y \cdot \supset F x) \cdot \supset F(\cup y)) \cdot \supset F z .
$$

But ' $x \subseteq z$ ' is redundant in view of ' $U y \subseteq z$ ' and ' $x \in y^{\prime}$ '. Dropping it, we see that the two clauses of the antecedent here follow respectively from the second and third premises of (3). So $F z$, q.e.d.

At this point we can apply Theorem I of [2], according to which, if a system $\mathfrak{S}$ contains extensionality, Aussonderung, and self- 
adjunction, viz., $(x)(\exists y)(y=x \cup\{x\})$, and ' $N O$ ' is an arbitrary predicate for which we can prove in $\mathbb{S}$ the law of transfinite induction, then we can prove in $\subseteq$ that if $N O(x)$ then $x$ is truly an ordinal. Accordingly, following the notation of [1], we have by extensionality, Aussonderung, self-adjunction, and power set that

$$
N O(x) \supset \cdot \cup x \subseteq x \cdot \text { Wellord } \Theta \uparrow x .
$$

The converse is pretty evident, for with the standard definition we expect to be able to prove most intuitively true statements about ordinals, and it is intuitively clear that all ordinals satisfy the condition in (2).

More formally, the converse of (4) follows immediately from T5 of [2]; for T5 shows that every genuine ordinal satisfies the condition in (2), granted extensionality, Aussonderung, and self-adjunction. Hence we have the

Theorem. Given extensionality, Aussonderung, self-adjunction, and power set, we can prove, with ' $N O$ ' defined by (2), that

$$
N O(x) \equiv \cdot \cup x \subseteq x \cdot \text { Wellord } \epsilon_{\uparrow}\lceil.
$$

The definition (2) is more elegant than the two definitions given in [2]. One of them, DI*, is like (2) except for the intrusion of an additional clause ' $z \subseteq x$ ' in the consequent. The two definitions in [2] have the advantage of not requiring the power-set axiom, but we feel it is more important to achieve economy in the definition. We do not know whether the theorem of this note remains true if we delete the power-set axiom. ${ }^{1}$

\section{REFERENCES}

1. W. V. Quine, Set theory and its logic, Harvard Univ. Press, Cambridge, Mass., 1963.

2. Hao Wang, $A$ theorem on definitions of the Zermelo-Neumann ordinals, Abstract 64T-36, Notices Amer. Math. Soc. 11 (1964), 137.

HARVARD UNIVERSITY

${ }^{1}$ Work for this note was supported by NSF grant GP-228. 